\title{
OBSERVATIONS OF THE PROGENITOR WIND OF SN 1987A
}

\author{
E.J. Wampler', A. Richichi ${ }^{2}$, D. Baade ${ }^{1}$ \\ 1. European Southern Observatory, Garching \\ 2. Arcetri Observatory, Firenze
}

As part of a general ESO program to monitor the spectrum of SN 1987A, we are collecting intermediate high resolution $\left(R=\lambda / \Delta \lambda \approx 2 \times 10^{4}\right)$ spectra of the supernova. By the end of the year 1987 it was possible to see narrow emission features due to [OIII] and hydrogen in spectra taken with the ESO Cassegrain echelle spectrograph (CASPEC) (Wampler and Richichi 1988, 1989). At that time, the velocities of these lines were about $286 \mathrm{~km} / \mathrm{sec}$ and their widths were about $14 \mathrm{~km} / \mathrm{sec}$ (FWHM). It is almost certain that these lines arise from a cloud of highly ionized gas that was first detected in ultraviolet spectra obtained by IUE in May 1987 (Fransson et al. 1989). This cloud is presumably a circumstellar shell that surrounded the progenitor of SN $1987 \mathrm{~A}$ and was ionized by the initial UV flash from the supernova explosion. In the ideal case of an expanding spherical shell that is large enough for light travel times to be important, one would expect the intensity of an emission line to increase and then decrease again as the light from the front of the shell fades. At the same time the velocity of the line would increase as the integrated light became dominated by light from the back (receding) hemisphere. The IUE light curve (Fransson et al. 1989) for the ultraviolet [NV] line was consistent with the photometric behavior expected from a nebula about 400 light days in diameter. At the distance of the LMC, one light year is equal to a span of one arc-second, and the photometric behavior of [NV] is consistent with the extent of the nebula measured by Wampler and Richichi (1989).

This paper gives the results of observations extending from July 181988 to Feb 27 1989. The July data are from a single echelle spectrogram obtained by Dr. George Wallerstein using the CTIO echelle spectrograph attached to their 4-meter telescope. The other spectra are from observations using CASPEC on the ESO 3.6-meter telescope at La Silla. These consist of a single spectrum obtained Aug 281988 and merged spectra obtained from short observing runs centered on Oct 1, 1988, Jan 1, 1989 and Feb 25, 1989. Because the spectra, and in particular the narrow lines, show 1ittle change over a period of a few days it is acceptable to group the spectra in this way.

The spectra were calibrated by comparing the CASPEC data with flux calibrated spectra obtained at much lower resolution with the ESO Boller and Chivens spectrograph attached to the ESO 1.5-meter telescope. This ratio of the CASPEC spectrum to a calibrated low resolution spectrum taken at about the same date was used to construct a smooth response function to rectify the CASPEC data. Fig. 1 shows a comparison of a rectified CASPEC spectrum overplotted on a low resolution calibrated 


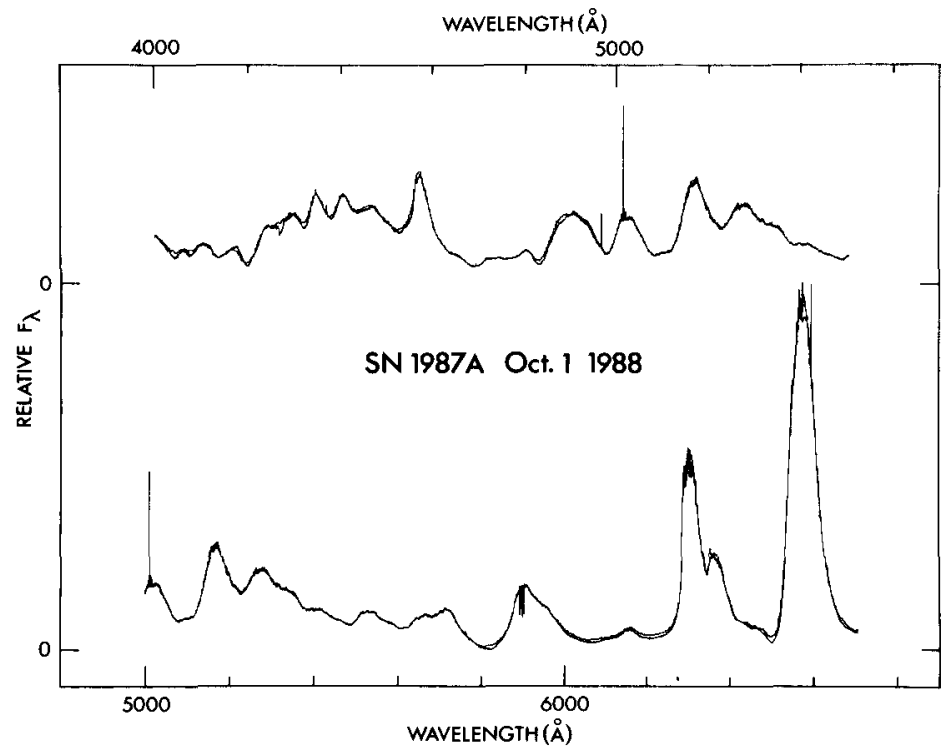

Fig. 1. A comparison between the low resolution flux calibrated spectrum and the CASPEC spectrum after it was corrected for the relative instrumental response function.

spectrum. Because the calculated response function is a very low frequency function the detailed agreement of the two spectra is an indication of the photometric accuracy of the two spectrographs. This procedure results in a relative calibration of the echelle data. An absolute calibration was then obtained by convolving the rectified echelle spectra with either a $\underline{B}$ or $\underline{V}$ filter function and comparing the derived "flux" with the appropriate $\underline{B}$ or $\underline{V}$ magnitude (Burki et al. 1989) for the date in question. Thus, although the calibration is not independent of these other data, it is sufficiently accurate for our purposes.

By the end of 1988 the Supernova had faded enough that many narrow nebular lines were visible. Fig. 2 shows the Feb 27, 1989 spectrum with some of the narrow lines identified. Table 1 lists the measured line intensities normalized to $H B=$

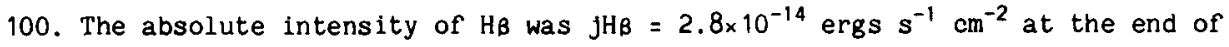
Dec 1987 and increased by about a factor of 3 by Feb 1989 (see Table 2 below). There is, in addition, a change in the temperature of the nebula and the level of ionization. An inspection of Table 1 shows that the [OIII] $\lambda \lambda 4959,5007$ lines have been decreasing in intensity relative to $H_{\beta}$ over the interval covered by these data. Thus, at the present time, the [OIII] lines have been nearly constant in strength. As noted above, [NV] peaked in intensity at about day 400. Therefore, the high ionization lines are weakening while the low ionization lines are increasing in strength. 


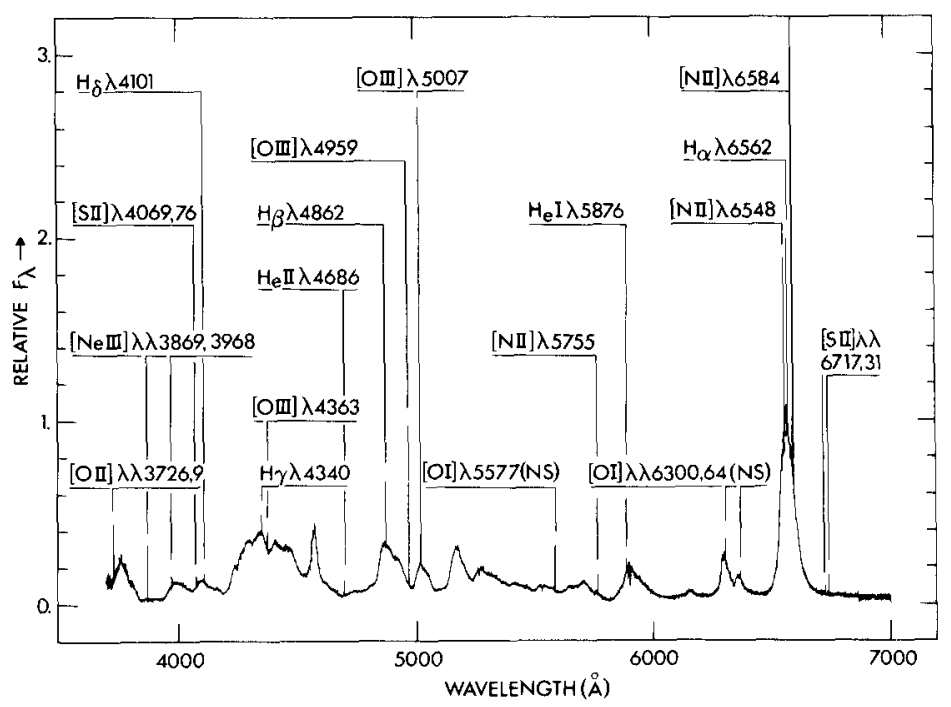

Fig. 2. The spectrum of SN $1987 \mathrm{~A}$ in Feb 1989. Narrow nebular lines are indicated.

Table 1: Relative Narrow Line Ratios

\begin{tabular}{|c|c|c|c|c|c|c|}
\hline Ion & $\lambda$ & $18 \mathrm{Jul}$ '88 & Aug' 88 & 1 oct' 88 & 1 Jan' 89 & 27 Feb' 89 \\
\hline [OII] & 3726 & $\cdots$ & $\ldots$ & $\cdots$ & $\cdots$ & 54 \\
\hline$[\mathrm{OII}]$ & 3729 & $\ldots$ & $\ldots$ & $\ldots$ & $\ldots$ & 23 \\
\hline [NeIII] & 3869 & $\ldots$ & $\ldots$ & $\ldots$ & $\ldots$ & 56 \\
\hline [NeIII] & 3968 & $\ldots$ & $\cdots$ & $\ldots$ & $\ldots$ & 13 \\
\hline $\mathrm{H} \varepsilon$ & 3970 & $\ldots$ & $\ldots$ & $\ldots$ & $\ldots$ & 5 \\
\hline [SII] & 4069 & $\ldots$ & $\ldots$ & 21 & 14 & 11 \\
\hline$[\mathrm{SII}]$ & 4076 & $\ldots$ & $\ldots$ & 13: & 6: & 4 \\
\hline $\mathrm{H} \delta$ & 4101 & $\ldots$ & $\ldots$ & 36 & 10.8: & 14 \\
\hline $\mathrm{Hy}$ & 4340 & $\ldots$ & 42 & 48 & 33 & 21 \\
\hline [OIII] & 4363 & 52 & $54:$ & 58 & 33 & 18 \\
\hline HeII & 4686 & $\ldots$ & 38 & 27 & 19 & 24 \\
\hline $\mathrm{HB}$ & 4862 & 100 & 100 & 100 & 100 & 100 \\
\hline [OIII] & 4959 & 350 & 384 & 280 & 213 & 221 \\
\hline [OIII] & 5007 & $\ldots$ & 1137 & 910 & 690 & 604 \\
\hline [NII] & 5755 & $\ldots$ & $\ldots$ & 28 & 32 & 23 \\
\hline $\mathrm{HeI}$ & 5876 & $\ldots$ & $\ldots$ & 24 & 25 & 15 \\
\hline$[\mathrm{OI}]$ & 6300 & $\ldots$ & $\ldots$ & $\ldots$ & $\ldots$ & 8: \\
\hline$[\mathrm{OI}]$ & 6364 & & $\ldots$ & $\ldots$ & $\ldots$ & 2.5 \\
\hline$[\mathrm{NII}]$ & 6548 & $\ldots$ & $\ldots$ & 243 & 263 & 220 \\
\hline $\mathrm{H} \alpha$ & 6562 & $\ldots$ & $\ldots$ & 393 & 363 & 324 \\
\hline [NII] & 6584 & $\ldots$ & $\ldots$ & 857 & 812 & 718 \\
\hline [SII] & 6717 & $\ldots$ & $\ldots$ & $\ldots$ & 17 & 12 \\
\hline [SII] & 6731 & $\ldots$ & $\ldots$ & $\ldots$ & 32 & 29 \\
\hline
\end{tabular}

Using the graphs published by Cantó et al. (1980) we find that the nebular density given by the [OII] $\lambda 3727$ doublet is about $1 \times 10^{4} \mathrm{~cm}^{-3}$ while the [SII] $\times 6724$ doublet gives a density of $4 \times 10^{4} \mathrm{~cm}^{-3}$. This is in good agreement with the density determined from the ultraviolet CIII] 1909 doublet (Fransson et al. 1989). Table 2 lists the temperature determinations for the nebula. The temperature is obtained from the equations: 


$$
\frac{j \lambda 6548+j \lambda 6583}{j \lambda 5755}=\frac{6.91 \exp \left[\left(2.50 \times 10^{4}\right) / \mathrm{T}\right]}{1+2.5 \times 10^{-3}\left(\mathrm{~N}_{\mathrm{e}} \mathrm{T}^{1 / 2}\right)}
$$

for the [NII] lines and

$$
\frac{j \lambda 4959+j \lambda 5007}{j \lambda 4363}=\frac{7.73 \exp \left[\left(3.29 \times 10^{4}\right) / \mathrm{T}\right]}{1+4.5 \times 10^{-4}\left(\mathrm{~N} \mathrm{e}^{\left./ \mathrm{T}^{1 / 2}\right)}\right.}
$$

for the [OIII] lines (Osterbrock 1989). The density was taken to be $3 \times 10^{4} \mathrm{~cm}^{-3}$ for all epochs; this might be a slight overestimate and could result in a slight underestimate of the electron temperature. The [OIII] temperature has decreased by about a factor of 2 since Dec 1987 (Wampler and Richichi, 1989). The [NII] temperature is significantly lower than that found from the [OIII] lines.

\section{Table 2: HB Flux and Temperature Determinations}

\begin{tabular}{lccccc} 
Date & $\begin{array}{c}\mathrm{JHB} \\
\left(\mathrm{ergs} \mathrm{s} \mathrm{s}^{-1}\right)\end{array}$ & $\frac{j 5007+j 4959}{j 4363}$ & T[OIII & $\frac{j 6584+j 6548}{j 5755}$ & \multirow{2}{*}{$\mathrm{T}[\mathrm{NII}]$} \\
JuI 18 1988 & $4.6 \times 10^{-14}$ & 27 & $2.5 \times 10^{4}$ & $\ldots$ & $\ldots$ \\
Aug 28 1988 & $6.4 \times 10^{-14}$ & 28 & $2.4 \times 10^{4}$ & $\ldots$ & $\ldots$ \\
Oct 1 1988 & $7.2 \times 10^{-14}$ & 21 & $3.0 \times 10^{4}$ & 39 & $1.11 \times 10^{4}$ \\
Jan 1 1989 & $7.4 \times 10^{-14}$ & 27 & $2.5 \times 10^{4}$ & 34 & $1.18 \times 10^{4}$ \\
Feb 27 1989 & $1.0 \times 10^{-13}$ & 39 & $1.9 \times 10^{4}$ & 41 & $1.09 \times 10^{4}$
\end{tabular}

It is worth noting that the Balmer decrement is slightly steeper than the value predicted for case $B$ radiative recombination. The observed decrement could be reconciled with the expected case $B$ value if one allows reddening corresponding to $E(B-V)=0.25 \mathrm{mag}$. This value is within the errors of the estimated reddening of the supernova (Fransson et al. 1989; Kirshner and Gilmozzi 1989; Wampler et al. 1987). There is, in addition, the possibility that there is dust mixed with the line emission region. In the $\lambda \lambda 6700-6800$ region the continuum level of the Supernova is very low (see fig. 2). Here the spectrum appears slightly wider and displaced to the west compared to the spectrum of the Supernova in spectral regions dominated by bright emission lines coming from the expanding supernova envelope. As the narrow emission lines from the circumstellar shell are also displaced slightly to the west these observations are compatible with dust mixed with the gas in the circumstellar shell. In the violet CaII absorption region, $\lambda \lambda 3800-3900$, where the Supernova spectrum is also faint, the continuum is narrow and undisplaced. This suggests that while the dust is reflecting the supernova light in the red, in the violet the early onset of CaII absorption in the Supernova spectrum extinguished the violet light that otherwise could be reflected from the dust in the $\lambda \lambda 3800-3900$ region.

The velacities of the lines have been changing. The velocity of the [OIII] lines is now about $290 \mathrm{~km} / \mathrm{sec}$, compared to $286 \mathrm{~km} / \mathrm{sec}$ in Dec 1987. We have sufficient data for the two epochs that we believe this difference is significant. 


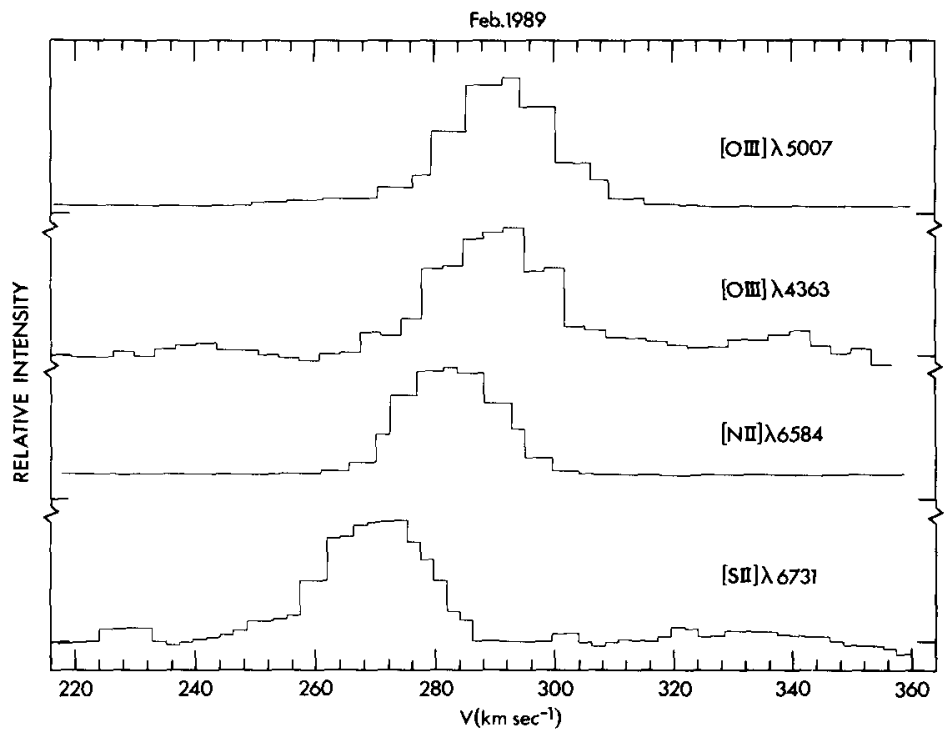

Fig. 3. The velocity profiles for lines of differing excitation.

The low ionization lines are at a lower velocity, about $260 \mathrm{~km} / \mathrm{sec}$. Fig. 3 shows the velocity structure found for several I ines in the Feb. 1989 spectrum. If the lowest velocities represent the velocity of the near side gas while the high velocities represent the back (receding) gas then the expansion velocity of the nebula is about $15 \mathrm{~km} / \mathrm{sec}$, somewhat larger than the expansion velocity estimated earlier (Wampler and Richichi 1989). These velocity differences, together with the differences in the gas temperatures determined from the [NII] or [OIII] lines, show that lines from different ionization states are coming from physically separate regions. Thus it is not possible to determine the relative abundances of the ions from these data.

Finally, the structure of the narrow emission line region is revealed in a series of spectra taken with CASPEC configured with a $15 \times 15$ arc second aperture. In this "slitless" mode the nebula is seen as a small " $C$ " shaped nebulosity about 1 arcsec in diameter and with a differential expansion of about $5 \mathrm{~km} / \mathrm{sec}$ across it. A similar profile is seen in [OIII], [NeIII], [NII] and [OII] lines. As the velocity gradient across the nebula in each line is smaller than the spread in velocity from ion to ion each ion must show only a portion of the true extent of the nebula.

We are indebted to the ESO SN $1987 \mathrm{~A}$ archive that is maintained by J. Danziger and R. Fosbury for the low resolution, flux calibrated spectra of SN 1987A that were used to rectify our high resolution echelle spectra. We also thank the Geneva observing team and $\mathrm{J}$. Danziger for their unpublished $\underline{B}, \underline{v}$ magnitudes which we used to determine the absolute calibration of our spectra. Finally, we thank J. Melnick for the Aug $1988 \mathrm{SN}$ spectrum and G. Wallerstein for the July 1988 one. 


\section{References}

Burki, G., Cramer, N., Burnet, M., Rufener, F., Pernier, B., and Richard, C. 1989, Astron. Astrophys., 213, L26.

Cantó, J., Elliott, K.H., Meaburn, J., and Theokas, A.C. 1980, MNRAS, $193,911$.

Fransson, C., Cassatella, A., Gilmozzi, R., Kirshner, R.P., Panagia, N., Sonneborn, G., and Wamsteker, W. 1989, Ap.J., 336, 429.

Kirshner, R.P., and Gilmozzi, R. 1989, "Observing SN 1987A with IUE", preprint.

Osterbrock, D.E. 1989, "Astrophysics of Gaseous Nebulae and Active Galactic Nuclei"

[University Science Books: Mill Valley, Calif.].

Wampler, E.J., Truran, J.W., Lucy, L.B., Höflich, P., and Hillebrandt, W. 1987,

Astron. Astrophys., 182, L51.

Wampler, E.J., and Richichi, A. 1988, The Messenger, No. 52, 14.

Wampler, E.J., and Richichi, A. 1989, Astron. Astrophys., in press.

\section{Discussion:}

TRIMBLE: What is this stuff?! That is, is it the same gas seen by IUE with a velocity even smaller than the IUE limit of $30 \mathrm{~km} \mathrm{sec}^{-1}$ ?

WAMPLER: Presumably yes, since it is enriched in nitrogen. The real velocity, of course, has a projection factor in it.

PARESCE: Can you tell from your data how the $\mathrm{C}$ structure you showed is actually oriented on the sky? Can the opening of the $\mathrm{C}$ be oriented towards the observer, for example?

WAMPLER: The answer to the first part is that the blue shifted side of the nebula is roughly North and the open part of the " $\mathrm{C}$ " is facing the East. The answer to the second part is, "not completely, but it may be partly open to the observer". 